01,05

\title{
Электрическое сопротивление редкоземельных металлов и их сплавов при высоких температурах: роль магнитного рассеяния
}

\author{
() А.Д. Ивлиев \\ Российский государственный профессионально-педагогический университет, \\ Екатеринбург, Россия \\ E-mail: ad_i48@mail.ru
}

Поступила в Редакцию 16 мая 2020 г.

В окончательной редакции 22 мая 2020 г.

Принята к публикации 26 мая 2020 г.

\begin{abstract}
Проанализированы результаты экспериментального исследования удельного электрического сопротивления редкоземельных металлов и их сплавов. Предложена процедура разделения вкладов в сопротивление. Установлено, что магнитный компонент электросопротивления в парамагнитной фазе снижается до нуля при нагреве. Отмечено, что современные модели явлений переноса не описывают данное явление.
\end{abstract}

Ключевые слова: редкоземельные металлы, сплавы, удельное электрическое сопротивление, магнитный компонент сопротивления.

DOI: 10.21883/FTT.2020.10.49900.110

\section{1. Введение}

Редкоземельные металлы (Р3М) - конденсированные фазы скандия, иттрия и лантаноидов [1]. Химические свойства РЗМ близки, что обусловлено однотипностью энергетических структур коллективизированных электронов. Тем не менее, обычно всю группу РЗМ разделяют на две подгруппы: иттриевую и цериевую. Металлы в пределах каждой из подгрупп проявляют наиболее близкие свойства. В данной статье будут рассмотрены металлы иттриевой подгруппы (то есть иттрий и тяжелые РЗМ от гадолиния по лютеций, исключая иттербий).

Сведения об удельном электрическом сопротивлении, представленные в настоящей статье, получены четырехзондовым методом на постоянном токе. Принцип работы типичной соответствующей экспериментальной установки описан, например, в [2].

В диапазоне высоких температур (то есть в парамагнитных областях выше температур Дебая) политермы электрического сопротивления чистых РЗМ [3] и их сплавов [4] однотипны. Скорость нарастания электрического сопротивления по мере нагрева снижается, а абсолютное значение удельного сопротивления оказывается достаточно высоким, превосходящим типичные значения этого параметра для прочих переходных металлов [3]. Важной особенностью политерм удельного электросопротивления является то, что они не образуют группу равноотстоящих друг от друга зависимостей, а представляют собой системы пересекающихся кривых. Данная особенность характерна как для чистых РЗМ, так и для исследованных сплавов.

Представляет интерес выяснение вопроса о том, какие механизмы рассеяния могут играть заметную роль в высокотемпературном электросопротивлении РЗМ и их сплавов? Энергетические структуры коллективизиро- ванных электронов в рассматриваемых металлах, как уже отмечалось, однотипны [5-10]. Поэтому различия в значениях удельных электросопротивлений связаны, в основном, с параметрами ионов [11-13]. Массы ионов при движении по ряду РЗМ от иттрия до лютеция монотонно увеличиваются вдвое. Этот факт должен привести к тому, что удельное электрическое сопротивление РЗМ, обусловленное рассеянием электронов на колебаниях решетки, должно постепенно снижаться при движении от легких металлов к тяжелым [11-13]. Для самых высоких температур, вблизи температур плавления, это так $[3,14,15]$. В области средних температур, однако, рекордсменом является гадолиний, обладающий наибольшим удельным электрическим сопротивлением среди Р3М [3]. Следовательно, кроме фононного, значительная роль в рассеянии электронов в рассматриваемой области температур принадлежит иному механизму, магнитномy [11].

Магнитные свойства ионов РЗМ значительно различаются. Они определяются $4 f$-электронами, состояния которых в конденсированной фазе обладают атомными свойствами, и не образуют энергетические зоны $[16,17]$. У паулевских парамагнетиков (иттрий и лютеций) магнитные моменты ионов равны нулю. У каждого из прочих ионов РЗМ имеется магнитный момент, связанный со спиновым и орбитальным вкладами.

Наибольшие орбитальное и полное квантовые числа у иона гольмия $[16,17]$. Поэтому, казалось бы, именно гольмий должен обладать наибольшим вкладом магнитного рассеяния в электросопротивление. Однако это не так. Кроме того, температура перехода гольмия в парамагнитное состояние оказывается в два раза ниже, чем у гадолиния, для которого эта температура максимальная среди РЗМ [3]. Ион гадолиния не обладает орбитальным магнитным моментом, но обладает наи- 
большим спиновым моментом [16,17]. Таким образом, именно спиновый магнетизм ионов обеспечивает высокое электросопротивление металлического гадолиния и наибольшую температуру перехода в парамагнитное состояние. Данный результат находится в согласии с моделью косвенного обмена, при котором магнитное взаимодействие ионов РЗМ обусловлено именно спином ионов $[6,7,16,17]$.

Кроме рассмотренных выше фононного и магнитного вкладов, в РЗМ и их сплавах присутствует еще и примесное рассеяние электронов. Других значимых механизмов рассеяния в рассматриваемом диапазоне температур нет [11-13]. В соответствии с правилом Маттиссена [11] полное удельное электросопротивление $\rho$ можно представить в виде

$$
\rho=\rho_{L}+\rho_{M}+\rho_{r},
$$

где $\rho_{L}, \rho_{M}, \rho_{r}$ соответственно фононный, магнитный и примесный компоненты. Для выяснения причин наблюдаемого поведения $\rho$ РЗМ и сплавов, необходимо оценить доли этих компонентов в полном сопротивлении. Иными словами, необходимо осуществить разделение вкладов в $\rho$.

\section{2. Методы разделения вкладов в удельное электрическое сопротивление}

Процедура разделения влияет на результат, и следовательно, от данной процедуры зависят физические выводы. Существуют три схемы разделения вкладов в $\rho$, используемые в настоящее время.

Первая схема основана на предположении, что фононное электрическое сопротивление любых металлов, в том числе и переходных, описывается законом Блоха [11]. В соответствии с ним $\rho_{L}$ при высоких температурах пропорционально температуре $\Theta$. Таким образом, прямая линия, проведенная от начала координат и параллельная высокотемпературному участку политермы $\rho(\Theta)$, отождествлялась с высокотемпературным вкладом фононов. Примесный компонент $\rho_{r}$ считался не зависящим от температуры, поэтому его значение определялось на основании знания отношения удельных электросопротивлений образца при комнатной температуре и при гелиевой температуре $(4.2 \mathrm{~K})$, поскольку при гелиевых температурах остальные вклады в $\rho$, кроме примесного, становятся ничтожно малы [11]. Затем по формуле (1) вычислялось $\rho_{M}$.

Рассматриваемая схема использовалась очень широко для переходных, металлов (см., например, $[3,6,16,18]$ ). Результаты, получаемые таким методом очевидны. В РЗМ величина $\rho_{M}$ оказывается значительно больше, чем $\rho_{L}$. Причем, учитывая, что политермы $\rho(\Theta)$ не прямые, а имеют отрицательную кривизну, $\rho_{L}$ зависит от того, для какой области температур на зависимости $\rho(\Theta)$ определялся „прямолинейный“ участок. Чем при больших температурах он определен, тем меньшее значение имеет $\rho_{L}(\Theta)$. Вклад, рассматриваемый как магнитный, обнаруживается во всех РЗМ. В частности, в немагнитном иттрии он в несколько раз больше, чем фононное сопротивление и связывается с неким электрон-электронным рассеянием [3]. При этом в РЗМ величины $\rho_{M}$ остаются значительными вплоть до температур плавления.

Существуют теоретические модели, позволяющие судить о температурной зависимости $\rho_{M}$ в парамагнитной фазе, в области высоких температур. Большая их часть указывает на то, что $\rho_{M}$ стремится к константе (т. е., имеет не нулевое значение) [6,16,19-21], или неограниченно возрастает пропорционально температуре [22]. Учитывая то, что как рассматриваемая методика разделения вкладов, так и модели магнитного рассеяния не обладают абсолютной точностью, между полученными экспериментальными результатами разделения вкладов и результатами теоретического рассмотрения противоречия нет. Это, однако, единственный положительный результат данного подхода.

Рассматриваемому методу разделения вкладов присущ недостаток, который является принципиальным - это использование модели Блоха для описания фононного сопротивления переходных металлов. Модель Блоха основана на использовании однополосной модели электронной проводимости, и в стандартном виде [11] не может быть использована для описания свойств переходных металлов, обладающих сложной электронной структурой $[11,12]$. Строго говоря, однополосная модель предназначена для щелочных металлов и не учитывает специфические процессы переноса заряда в многополосных системах [23]. Именно поэтому при обработке результатов возникают большие по величине вклады в сопротивление, которые, в рамках однополосной модели и рассматриваемой схемы разделения, приписываются магнитному рассеянию. Данные вклады появляются не только в иттрии, но во всех РЗМ - паулевских парамагнетиках. Такие выводы противоречит имеющимся фундаментальным результатам теории явлений переноса в твердых телах [11-13].

Результаты анализа электросопротивления, проведенные по первой схеме, не позволяют понять и ряд других особенностей политерм $\rho$ РЗМ и сплавов, которые будут рассмотрены ниже, при описании процесса обработки экспериментальных данных (раздел 3). Иными словами, рассматриваемую первую схему разделения вкладов в удельное электросопротивление РЗМ (да и вообще, переходных металлов) следует признать ошибочной. Ошибочны, следовательно, и полученные с ее помощью выводы.

Вторая схема, в соответствии с которой можно разделить вклады в $\rho$, основана на том, что электросопротивление РЗМ - паулевских парамагнетиков, содержит только примесный и фононный компоненты. В частности, для рассматриваемых РЗМ, паулевскими 
парамагнетиками являются иттрий и лютеций. Следовательно, вычитая из $\rho$ магнитных РЗМ $\rho$ иттрия или лютеция, можно выделить магнитный вклад в сопротивление $[24,25]$. Физически данный подход представляется правильным. Выделенная таким образом магнитная составляющая удельного электросопротивления оказывается убывающей при возрастании температуры [24]. Недостатком рассматриваемого подхода является то, что $\rho$ иттрия при высоких температурах оказывается больше, чем у других РЗМ, и разность удельных сопротивлений становится отрицательной (то есть стремится к отрицательной константе при повышении температуры). В случае, когда вычитается $\rho$ лютеция, эта разность стремится к положительной константе. Иными словами, данный метод разделения вкладов, несмотря на физическую прозрачность, оказывается неоднозначным.

Третья схема разделения вкладов по физической сущности и по своим основным результатам оказывается близка ко второй схеме, однако в данном случае используется перенормировка удельных сопротивлений РЗМ, благодаря чему неоднозначность исключается. Следует отметить, что вторая и третья схемы разделения вкладов применимы только к материалам, имеющим подобные энергетические структуры внешних электронных состояний атомов, и следовательно, коллективизированных электронов в конденсированной фазе. Такими веществами среди элементов Периодической таблицы Д.И. Менделеева оказываются только РЗМ.

Суть метода разделения вкладов в электросопротивление чистых РЗМ изложена, например, в [26]. В основе ее лежит модель двухполосной проводимости Мотта [27] простейшая модель, предназначенная для описания кинетических явлений в переходных металлах. Отметим, что реально, энергетическая структура коллективизированных электронов в РЗМ более сложная, чем в используемой модели, однако такой упрощенный подход позволяет проследить за основными рассматриваемыми проблемами в аналитической форме, что удобно. Итак, модель рассматривает две энергетические полосы $s$ и $d$-электронов. Первая $s$-полоса имеет сравнительно низкую и слабо изменяющуюся в зависимости от энергии $W$ плотность электронных состояний $N_{s}(W)$. Вследствие этого, $s$-электроны обладают малой эффективной массой [13]. Вторая $d$-полоса является узкой, плотность состояний электронов в ней $N_{d}(W)$ выше, чем в $s$-полосе, и изменяется она в зависимости от энергии электронов значительно более сильно, чем $N_{s}(W)$. Вследствие этого, $d$-электроны имеют бо́льшую эффективную массу, чем $s$-электроны.

Наличие двух энергетических полос электронов позволяет полную проводимость вещества $\sigma=1 / \rho$ представить в виде суммы проводимостей каждой из полос

$$
\sigma=1 / \rho=\sigma_{s}+\sigma_{d},
$$

где $\sigma_{s}$ и $\sigma_{d}-$ проводимости, обусловленные движением $s$ - и $d$-электронов соответственно. Не претендуя на количественную точность, упростим это соотношение, предположив, что вследствие разных величин эффективных масс $\sigma_{s} \gg \sigma_{d}[12]$. Тогда

$$
\sigma=1 / \rho \approx \sigma_{s}=1 / \rho_{s}
$$

где $\rho_{s}$ - удельное электрическое сопротивление проводника, создающееся за счет $s$-электронов. Величина удельного электрического сопротивления, а, следовательно, и проводимости, ограничивается процессами рассеяния носителей [13]. Вне зависимости от физической природы рассеяния, изменение состояния $s$-электрона может происходить двумя путями: либо электрон остается в $s$-полосе (ss-переход, вклад $\left.\rho_{s s}\right)$, либо переходит в $d$-полосу $\left(s d\right.$-переход, вклад $\left.\rho_{s d}\right)$. Следовательно,

$$
\rho \approx \rho_{s}=\rho_{s s}+\rho_{s d} .
$$

Вероятности переходов определяются, в частности, плотностями электронных состояний вблизи химического потенциала $\eta$ (то есть, при $W=\eta$ ) [13]. По оценке Займана [11]

$$
\rho_{s d} \approx \rho_{s s} N_{d}(\eta) / N_{s}(\eta) .
$$

Таким образом [25],

$$
\begin{aligned}
\rho & \approx \rho_{s s}+\rho_{s d} \approx \rho_{s s}+\rho_{s s} N_{d}(\eta) / N_{s}(\eta) \\
& =\rho_{s s}\left[1+N_{d}(\eta) / N_{s}(\eta)\right],
\end{aligned}
$$

где $\rho_{s s}$ - величина удельного электрического сопротивления, рассчитанная для одной $s$-полосы. Если выражение (6) используется для описания фононной составляющей $\rho_{L}$, то в качестве $\rho_{s s}$ следует взять, например, формулу Блоха [12]:

$$
\rho_{s s}(\theta)=\frac{\left(3 \pi^{2}\right)^{1 / 3} \pi^{3} \hbar^{3}}{4 e^{2} k} \frac{N^{1 / 3}}{n_{a}^{2 / 3} \theta_{R}^{2} m_{A} V^{1 / 3}} \theta,
$$

где $n_{a}$ - число электронов проводимости на атом, $m_{A}-$ масса иона, $N$ - число ионов в объеме $V, \theta_{R}-$ кинетическая температура Дебая для электросопротивления, $k$ - постоянная Больцмана, $e-$ заряд электрона, $\Theta-$ температура.

На основании (6) и (7) запишем

$$
\rho_{L}=Z(\theta) \frac{\gamma^{1 / 3}}{\theta_{R}^{2}} \frac{\theta}{M^{4 / 3}},
$$

где $\gamma-$ плотность, $M$ - молярная масса проводника, $Z(\Theta)$ - прочие параметры, включая зависящую от температуры плотность состояний $d$-электронов. Функция $Z(\Theta)$ включает в себя такие параметры, которые оказываются одинаковыми для всех рассматриваемых РЗМ. Значения параметров $\gamma, M$ и $\Theta_{R}$ для чистых РЗМ содержатся в справочнике [3].

Функцию $Z(\Theta)$ можно рассчитать на основании удельного фононного электросопротивления иттрия. Зная 
ее, можно затем на основании табличных данных о плотности, молярной массе и кинетической температуре Дебая лютеция, рассчитать удельное фононное электросопротивление лютеция. Полученное сопротивление, как показывает опыт, в пределах погрешности измерений $\rho$ совпадает с экспериментальным результатом [26]. Это доказывает, что функция $Z(\Theta)$ является универсальной для всех рассматриваемых РЗМ. Таким образом, при известной функции $Z(\Theta)$ может быть рассчитано удельное фононное электросопротивление $\rho_{L}$ любого рассматриваемого РЗМ.

Примесное электросопротивление оценивается, как обычно, по соотношению сопротивлений при комнатной температуре и при $4.2 \mathrm{~K}$. В конечном итоге на основании (1) рассчитывается магнитный вклад для каждого металла.

В том случае, когда речь идет о сплавах РЗМ (а это твердые растворы), вычисления $\rho_{L}$ проводятся по той же формуле (8). При этом возникает проблема оценки параметров сплавов, таких как $\gamma, M$ и $\Theta_{R}$, поскольку в литературе данная информация отсутствует. В первом приближении значения этих параметров могут быть оценены, исходя из концентраций компонентов [28]. Для сплавов $\mathrm{Y}-\mathrm{Ho} \mathrm{(иттрий-гольмий)} \mathrm{получим}$

$$
\begin{gathered}
M=(1-x) M_{\mathrm{Ho}}+x M_{\mathrm{Y}}, \\
\Theta_{R}=(1-x) \Theta_{R, \mathrm{Ho}}+x \Theta_{R \mathrm{Y}}, \\
\gamma=(1-n) \gamma_{\mathrm{Ho}}+n \gamma_{\mathrm{Y}}, \\
x=\frac{n}{n+\frac{(1-n) M_{\mathrm{Y}}}{M_{\mathrm{Ho}}}},
\end{gathered}
$$

где $x$ - концентрация иттрия (атомные доли), $n-$ концентрация иттрия (массовые доли), индексы у $\gamma, M$ и $\Theta_{R}$ означают металл, чей параметр используется в формулах. Таким образом, формула (8) оказывается пригодной для расчета фононного электрического сопротивления не только чистых РЗМ, но и их сплавов.

Магнитный компонент удельного электрического сопротивления сплавов может быть рассчитан на основании знания $\rho_{M}$ магнитного металла сплава (в рассматриваемом случае это гольмий). Теория показывает, что в парамагнитной фазе величина этого вклада в удельное сопротивление оказывается пропорциональна концентрации магнитных ионов [9], таким образом, имея в виду систему $\mathrm{Y}-\mathrm{Ho}$, запишем

$$
\rho_{M,(1-x)}=(1-x) \rho_{M, \mathrm{Ho}} .
$$

В дальнейшем на основании знания $\rho_{L}$ и $\rho_{M}$ по формуле (1) может быть рассчитана величина примесного удельного электрического сопротивления сплавов.

\section{3. Обработка экспериментальных результатов}

Рассмотрим результаты исследований удельного электросопротивления РЗМ и сплавов $\mathrm{Y}-\mathrm{Ho}$, представленные в $[3,4,14,15,26,28]$. В соответствии с рассмотренной выше третьей схемой разделения вкладов, по формуле (8) были рассчитаны фононные удельные электрические сопротивления $\rho_{L}$ чистых РЗМ, оценены примесные сопротивления $\rho_{r}$, и затем вычислены магнитные компоненты $\rho_{M}$. Полученные зависимости, естественно, имели подобный вид, и отличались лишь количественно. На рис. 1 представлены результаты расчетов для $\mathrm{Gd}$, имеющего наибольшее магнитное сопротивление.

Из графиков видно, что по мере нагрева фононный компонент сопротивления $\rho_{L}$ возрастает и приближается к полному $\rho$, а магнитный компонент $\rho_{M}$ стремиться к нулю. Такой ход температурных зависимостей является общим для РЗМ и их сплавов.

На рис. 2 показаны магнитные компоненты удельного электросопротивления для РЗМ, имеющих наибольшие

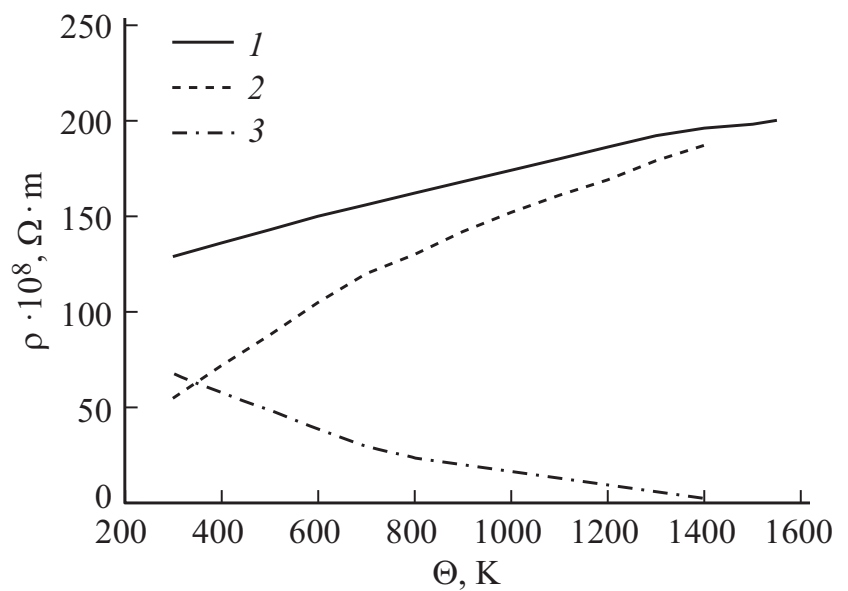

Рис. 1. Удельное электрическое сопротивление $\mathrm{Gd}$ и его компоненты $\left(1-\rho, 2-\rho_{L}, 3-\rho_{M}\right)$.

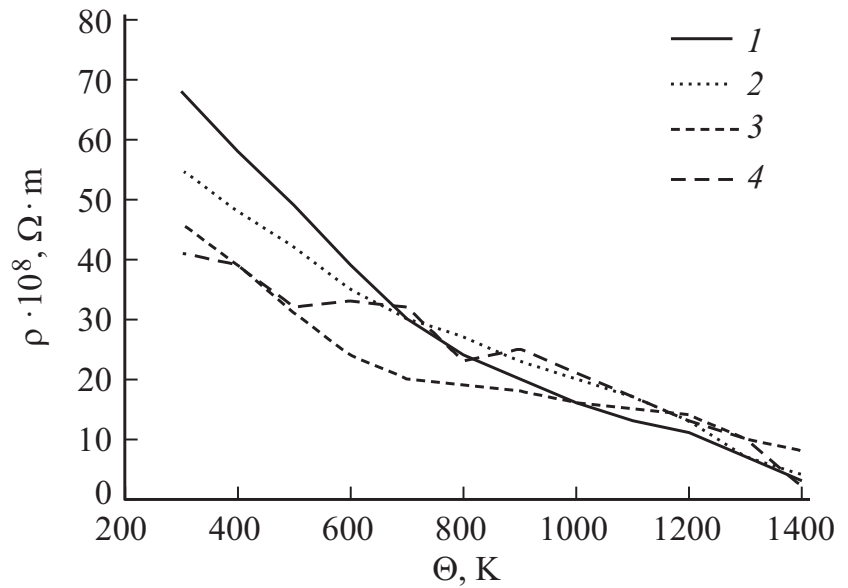

Pис. 2. Магнитный компонент сопротивления $\rho_{M} \mathrm{Gd}, \mathrm{Tb}$, Dy и Но. $(1-\mathrm{Gd}, 2-\mathrm{Tb}, 3-\mathrm{Dy}, 4-\mathrm{Ho})$. 


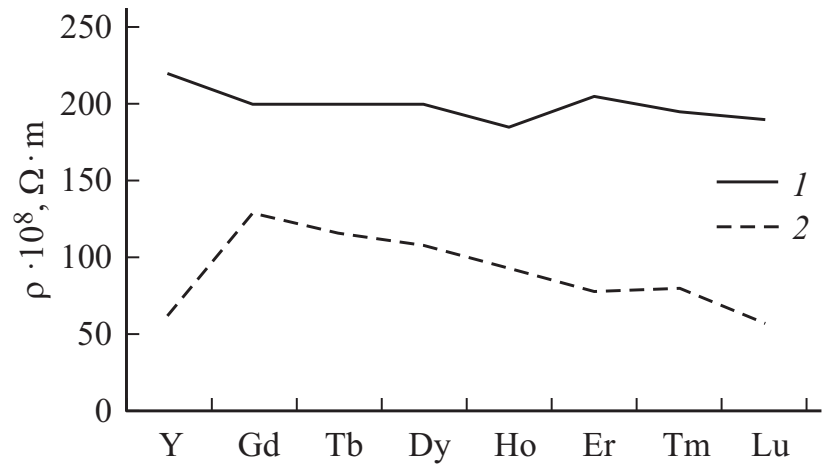

Рис. 3. Полное удельное электрическое сопротивление РЗМ иттриевой подгруппы $(1-300 \mathrm{~K}, 2-1500 \mathrm{~K})$.

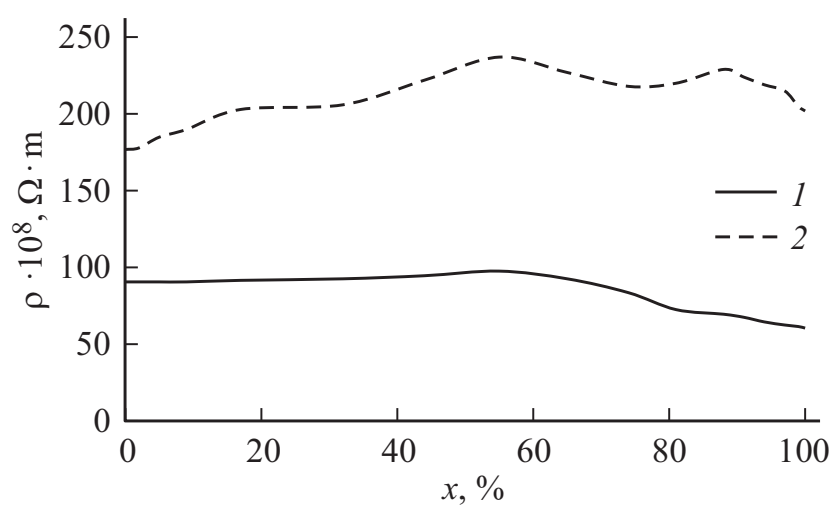

Рис. 4. Концентрационные зависимости полного удельного электрического сопротивления сплавов Ү-Но $(1-300 \mathrm{~K}$, $2-1400 \mathrm{~K})$.

значения $\rho_{M}$, это Gd, Tb, Dy и Но. Аналогичные результаты и для сплавов. Таким образом, при температурах выше $1000 \mathrm{~K}$ роль магнитного рассеяния в рассматриваемых веществах становится мала.

Данный вывод следует и из рассмотрения зависимостей величин $\rho$ РЗМ для двух значений температуры: комнатной $(300 \mathrm{~K})$ и высокой $(1500 \mathrm{~K})$, которые представлены на рис. 3 .

При комнатной температуре величина магнитного компонента сопротивления велика. Из-за этого $\rho$ магнитных металлов существенно превышают значения удельных сопротивлений паулевских парамагнетиков Y и Lu. Однако, при высоких температурах ситуация изменяется. Как видно, удельное сопротивление Y становится наибольшим, а по мере приближения к $\mathrm{Lu}$ величина $\rho$ снижается. Этот результат находится в согласии с моделью (8), которая описывает фононный вклад. Роль магнитного вклада перестает быть заметной.

Аналогичный вывод можно сделать из анализа концентрационных зависимостей $\rho$ сплавов $\mathrm{Y}-\mathrm{Ho}$, показанных на рис. 4. При низких температурах $(\Theta=300 \mathrm{~K}) \rho$ Но $(x=0)$ больше, чем $\rho$ Y. Причина этого - магнитное рассеяние. По мере роста концентрации Y (по мере роста $x) \rho$ остается практически неизменным до $x=60 \%$.
При этом повышение концентрации иттрия и падение доли магнитного сопротивления (12) компенсируются ростом примесного сопротивления, обусловленного увеличением структурного беспорядка в сплаве. Как показывает опыт, между этими влияниями на электросопротивление имеется баланс, и потому $\rho$ практически не изменяется.

При $x>60 \%$ падение сопротивления происходит. Это связано как с тем, что снижается магнитное рассеяние, так и с тем что, снижается примесное рассеяние. Максимум примесного рассеяния, как показывает анализ [28], приходится на середину концентрационной зависимости. Таким образом, при температуре $300 \mathrm{~K}$ роль магнитного рассеяния заметна. При температуре $1400 \mathrm{~K}$ ситуация иная. Сопротивление Но меньше, чем сопротивление Y. При движении по концентрационной зависимости наблюдается только эффект от структурного беспорядка (максимум сопротивления вблизи $x=50 \%$, правило Нордгейма [11]). Влияние магнитного рассеяния не прослеживается.

Наблюдаемое экспериментально пересечение политерм $\rho$ чистых Р3М $[3,14,15]$ и их сплавов [4,28] как раз и вызвано тем, что по мере роста температуры снижается величина $\rho_{M}$ практически до нуля, и основным рассеивающим фактором становятся колебания решетки.

Рассмотренные особенности концентрационных и температурных зависимостей удельных электросопротивлений РЗМ и их сплавов не находят объяснения в том случае, если принять, что $\rho_{M}$ при высоких температурах является постоянной величиной (как это следует из первой схемы разделения вкладов).

Таким образом, имеющийся экспериментальный материал позволяет утверждать, что магнитное сопротивление в парамагнитных РЗМ и их сплавах дает заметный вклад в $\rho$ только при средних температурах. При высоких температурах этого вклада практически нет.

\section{4. Обсуждение полученных результатов}

Проблема, возникшая из рассмотрения температурной зависимости $\rho_{M}$ РЗМ и их сплавов, заключается в том, что на сегодняшний день отсутствует физическая модель, объясняющая снижение магнитного электросопротивления парамагнитных металлов при нагреве. Следует отметить, что и при низких температурах, когда магнитные структуры РЗМ находятся в упорядоченном состоянии, модели не дают достаточно точного описания температурной зависимости $\rho_{M}(\Theta)$.

Для иллюстрации этого лучше всего провести анализ свойств гадолиния, поскольку его $\rho_{M}$ наибольшее, и потому относительная погрешность оценки минимальна. Процесс выделения $\rho_{M}$ проведем по третьей схеме, вычисляя $\rho_{L}$ по формуле (8). Для низкотемпературной области воспользуемся литературными данными для $\rho$ 


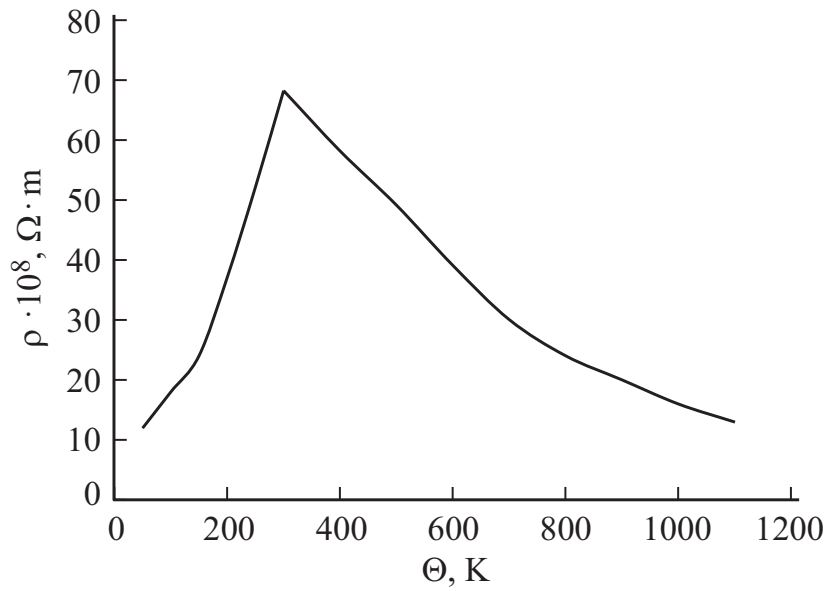

Рис. 5. Магнитный компонент электросопротивления $\rho_{M} \mathrm{Gd}$ в ферромагнитной и парамагнитной областях температур.

из справочника [3]. Соответствующий результат показан на рис. 5.

Видно, что $\rho_{M}$ это дополнительный вклад в $\rho$, обеспечивающий превышение величины сопротивления над фононным и примесным вкладами. Максимальная величина $\rho_{M}$ соответствует температуре Кюри $\Theta_{\mathrm{C}}$ [3]. Закономерности, определяющие изменение сопротивления при нагреве, неодинаковы для ферромагнитной и парамагнитной областей. Неодинаковы, естественно, и физические причины, ответственные за рост и снижение $\rho_{M}$. Аппроксимация полученных результатов дает следующие соотношения:

для $\Theta<\Theta_{\mathrm{C}}: \quad \rho_{M}=14 \cdot 10^{-11} \cdot \Theta^{3 / 2}, \Omega \cdot \mathrm{m}$,

для $\Theta>\Theta_{\mathrm{C}}: \rho_{M}=145 \cdot 10^{-8} \exp \left(-2.2 \cdot 10^{-3} \Theta\right), \Omega \cdot \mathrm{m}$.

Опыт показывает, что в ферромагнитной фазе рост $\rho_{M}$ происходит по степенному закону (13). Такой ход политермы, в целом, соответствует имеющимся теоретическим представлениям о роли магнитного рассеяния $[6,16]$. Однако, показатель степени $3 / 2$ установлен только для „экзотического случая“: рассеяния электронов на двухмерных спиновых волнах вблизи границ доменов [16]. Достаточно общего решения этой задачи, позволяющего оценить $\rho_{M}$ ферромагнитного металла в рамках модели многополосной проводимости, до сих пор нет.

В области высоких температур $\Theta>\Theta_{\mathrm{C}}$ опыт показывает, что величина магнитного сопротивления уменьшается практически до нуля. Убывание происходит по экспоненциальному закону (14). На сегодняшний день нет моделей, опирающихся на „первые принципы“, и позволяющие понять данный факт. Как уже отмечалось выше, теоретические расчеты дают иные результаты: $\rho_{M}$ либо остается неизменным, либо даже возрастает при нагреве в парамагнитной области. Парамагнитная фаза рассматривается как область наивысшего магнитного беспорядка в системе, благодаря чему в большинстве моделей $\rho_{M}$ достигает максимума при $\Theta_{\mathrm{C}}$ и далее при повышении температуры остается неизменным. Таким образом, имеющиеся модели противоречат опыту.

Вместе с тем, постоянство $\rho_{M}$ при высоких температурах является следствием приближений, сделанных при проведении вычислений. В частности, рассмотрим классический результат оценки $\rho_{M}[6]$. В парамагнитном состоянии величина сопротивления определяется функцией де Жена $G$ :

$$
\begin{gathered}
G=\left(g_{J}-1\right)^{2} J(J+1), \\
\rho_{M}=\frac{3 \pi N m_{e}^{*}}{8 \hbar e^{2} \eta V} A_{0}^{2} G,
\end{gathered}
$$

где $g_{J}$ - фактор (множитель) Ланде [6], $J-$ полное квантовое число иона РЗМ [17], $A_{0}$ - параметр обмена (обменный интеграл), $N$ - число ионов в объеме $V$, $m_{e}^{*}$ - эффективная масса электрона проводимости, $\eta-$ химический потенциал (уровень Ферми) электронов проводимости, $e$ - заряд электрона. В выражении (16) все представленные величины являются константами, в том числе и обменный интеграл $A_{0}$. Реально, однако, этот параметр косвенного обмена зависит от взаимного расстояния между ионами металла. Как известно, спиновая плотность электронов проводимости в зависимости от расстояния при косвенном обмене является осциллирующей функцией $[6,17,29]$ (подобно тому, как осциллирует плотность электрического заряда экранированного потенциала примеси [30]). Естественно, что и величина обменного интеграла должна оказаться зависящей от расстояния между ионами, и следовательно, от температуры. Это обстоятельство должно привести К температурному изменению $\rho_{M}$. К сожалению, последовательные расчеты, учитывающие непостоянство обменного интеграла, не произведены. Не рассмотрены и процессы многополосного рассеяния при оценке $\rho_{M}$.

Хотелось бы отметить, что при оценке $\rho_{M}$ не только РЗМ, но и других переходных металлов и сплавов, также наблюдаются противоречия между экспериментом и модельными представлениями [31].

Таким образом, в настоящее время задача адекватного описания магнитного компонента рассеяния электронов в переходных, в том числе редкоземельных металлах, в широком диапазоне температур не решена. Проблема эта принадлежит к разряду фундаментальных, ее решение необходимо для выяснения физики магнетизма и физики явлений переноса в переходных металлах при высоких температурах.

\section{5. Выводы}

Экспериментальное исследование удельного электрического сопротивления РЗМ и их сплавов позволило установить, что магнитный компонент электрического 
сопротивления в парамагнитной фазе в твердом состоянии снижается по мере нагрева.

Методы теоретического описания магнитного рассеяния, разработанные к настоящему времени, противоречат данному результату.

\section{Финансирование работы}

Исследование выполнено при финансовой поддержке РФФИ в рамках научных проектов № 11-08-00275 и 14-08-00228.

\section{Конфликт интересов}

Авторы заявляют, что у них нет конфликта интересов.

\section{Список литературы}

[1] Н.Л. Глинка. Общая химия / Под ред. А.И. Ермакова. Интеграл-Пресс, М. (2000). 728 с.

[2] М.Ю. Черноскутов, А.Д. Ивлиев, В.В. Мешков, А.О. Самойлов, А.С. Соснин. Измерительная техника 5, 60 (2018).

[3] В.Е. Зиновьев. Теплофизические свойства металлов при высоких температурах. Металлургия, М. (1989). 384 с.

[4] М.Ю. Черноскутов, А.Д. Ивлиев, В.В. Мешков. В сб.: XV Рос. конф. (с междунар.участием) по теплофизическим свойствам веществ (РКТС-15) и научная школа для молодых ученых. Тезисы докл. (Москва, 15-17 октября 2018 г.) Янус-К, М. (2018). С. 90.

[5] Handbook on the Physics and Chemistry of the Rare Earths / Ed. K.A. Gschneider Jr, L. Eyring. North-Holland, Amsterdam (1978). 1: Metals.

[6] К. Тейлор, М. Дарби. Физика редкоземельных соединений. Мир, М. (1974). 375 c.; [K.N.R. Taylor, M.I. Darby. Physics of Rare Earth Solids. Chapman and Hall Ltd., London. (1972)].

[7] C.А. Никитин. Магнитные свойства редкоземельных металлов и их сплавов. МГУ, М. (1989). 248 с.

[8] Y. Wang, M.Y. Chou. Phys. Rev. B 44, 10339 (1991).

[9] R.I.R. Blyth, S.D. Barrett, S.S. Dhesi, R. Cosso, N. Heritage, A.M. Begley, R.G. Jordan. Phys. Rev. B 44, 5423 (1991).

[10] В.В. Немошкаленко, В.Н. Антонов, В.Г. Алешин. Докл. АН СCCР 228, 837 (1976).

[11] Дж. Займан. Электроны и фононы. Изд-во ИЛ, М. (1962). 488 c.; [J.M. Ziman. Electrons and Phonons. Oxford at the Clarendon Press (1960). 397 p.].

[12] Ф. Блатт. Физика электронной проводимости в твердых телах. Мир, М. (1971). 470 c.; [F.J. Blatt. Physics of Electronic Conduction in Solids. McGraw-Hill Book Company (1968)].

[13] Н.Ашкрофт, Н. Мермин. Физика твердого тела. Мир, М. (1979). 1. 399 c.; 2.422 c.); [N.W. Ashcroft, N.D. Mermin. Solid State Physics. Holt, Rinehart and Winston, N.Y. (1976)].

[14] А.Д. Ивлиев. Высокотемпературные теплофизические свойства твердых редкоземельных металлов. Дис. докт. физ. мат. наук. Уральский горный институт им. В.В. Вахрушева, Екатеринбург (1991). 455 с.

[15] А.Н. Поздеев, А.Д. Ивлиев, А.А. Куриченко, Е.3. Ривман, Н.И. Морева. ФММ 9, 85 (1990).

[16] С.В. Вонсовский. Магнетизм. Наука, М. (1971). 1032 с.
[17] Р. Уайт. Квантовая теория магнетизма. Мир, М. (1985). 303 c.) ; [R.M. White. Quantum Theory of Magnetism. Springer-Verlag, Berlin Heidelberg N.Y. (1983)].

[18] В.Ю. Ирхин, Ю.П. Ирхин. Электронная структура, физические свойства и корреляционные эффекты в $d$ - и $f$-металлах и их соединениях. УрО РАН, Екатеринбург. (2004). $472 \mathrm{c}$.

[19] A. Watabe, T. Kasuya. J. Phys. Soc. Jpn 26, 64 (1969).

[20] R. Jullien, M.T. Beal-Monod, B. Coqblin. Phys. Rev. B 9, 1441 (1974).

[21] В.И. Гребенников, Ю.И. Прокопьев. ФММ 60, 213 (1985).

[22] В.Т. Швец. ФТТ 33, 261 (1991).

[23] Ю.Ю. Циовкин, Л.Ю. Вишнеков, А.Н. Волошинский. ФММ 8, 76 (1991).

[24] H.-J. Güntherodt, E. Hauser, H.U. Künzi. Proc. 3rd Int. Conf. on Liquid Metals. Bristol, 1976; Inst. Phys. Conf. Ser. N 30, Bristol and London. (1977) P. 324.

[25] A.T. Burkov, M. Vedernikov, E. Grats. Physica B 176, 263 (1992).

[26] А.Д. Ивлиев. ФММ 75, 9 (1993).

[27] N.F. Mott. Proc. Phys. Soc. 47, 571 (1935).

[28] А.Д. Ивлиев, М.Ю. Черноскутов, В.В. Мешков, А.А. Куриченко, Н.И. Морева. В кн.: V Междунар. научнотехн. конф. „Современные методы и средства исследования теплофизических свойств веществ“. 23-24 мая 2019 г. Сб. трудов. ИТМО, СПб (2019). С. 8. eLIBRARY.RU https://www.elibrary.ru/item.asp?id $=42140558$

[29] К.П. Белов. Редкоземельные магнетики и их применение. Наука, М. (1980). 239 с.

[30] Дж. Займан. Принципы теории твердого тела. Мир, М. (1974). 472 c.); [J.M. Ziman. Principles of the Theory of Solids. Cambridge at the University Press. (1972)].

[31] А.Д. Ивлиев, Ю.В. Глаголева. ФТТ 53, 1106 (2011).

Редактор К.В. Емцев 